\title{
RNA-Seq Reveals Hawthorn Tree as a New Natural Host for Apple Necrotic Mosaic Virus, Possibly Associated with Hawthorn Mosaic Disease
}

\author{
Fei Xing, ${ }^{1,2}$ Wanying Hou, ${ }^{1,3}$ Sebastien Massart, ${ }^{3}$ Dehang Gao, ${ }^{4}$ Wenhui Li, ${ }^{5}$ Mengji Cao, ${ }^{6}$ Zhixiang Zhang, ${ }^{1}$ Hongqing Wang, ${ }^{4}$ \\ and Shifang $\mathbf{L i}^{1,2, \dagger}$ \\ ${ }^{1}$ State Key Laboratory for Biology of Plant Diseases and Insect Pests, Institute of Plant Protection, Chinese Academy of Agri- \\ cultural Sciences, Beijing 100193, China \\ ${ }^{2}$ Environment and Plant Protection Institute of Chinese Academy of Tropical Agricultural Sciences (CATAS), Haikou 571101, \\ China \\ ${ }^{3}$ Plant Pathology Laboratory, TERRA, Gembloux Agro-Bio Tech, University of Liège, Gembloux, Belgium \\ ${ }^{4}$ College of Horticulture, China Agricultural University, Beijing 100193, China \\ ${ }^{5}$ National Fruit Tree Germplasm Repository, Xinjiang Academy of Agricultural Sciences, Luntai 841600, China \\ ${ }^{6}$ National Citrus Engineering Research Center, Citrus Research Institute, Southwest University, Chongqing 400712, China
}

\begin{abstract}
Apple mosaic disease is widespread in the major apple-producing areas in China and is frequently associated with the presence of the newly identified Apple necrotic mosaic virus (ApNMV), belonging to subgroup 3 of Ilarvirus genus in the family of Bromoviridae. Mosaic symptoms were also observed in a hawthorn tree. Deep sequencing revealed the hawthorn tree with mosaic symptom was infected by ApNMV, which was confirmed by RT-PCR. The complete nucleotide sequences of RNA1 $(3,378 \mathrm{nt})$, RNA2 (2,778 nt), and RNA3 (1,917 nt) of ApNMV from the hawthorn were obtained, sharing 93.8 to $96.8 \%, 89.7$ to $96.1 \%$, and 89.8 to $94.6 \%$ nucleotide identities with those from apples and crabapples, respectively. Two hypervariable regions were found, which showed 59.2 to $85.7 \%$ and 64.0 to $89.3 \%$ sequence identities at position

hawthorns to apple trees, with severe chlorosis, yellowing, mosaic, curling, and necrosis. In addition, a total of 11,685 hawthorn trees were surveyed for the incidence of mosaic disease from five provinces in China, and only six were found showing typical mosaic symptoms. A total of 145 individual trees (six symptomatic, 68 asymptomatic, and 71 other symptoms) were tested for the presence or absence of ApNMV by RTPCR. Among them, six symptomatic, four asymptomatic, and 10 other symptomatic trees tested positive for ApNMV. Taken together, these results demonstrated that the hawthorn tree was identified as a new natural host for ApNMV with a relatively low frequency $(13.8 \%, 20$ out of 145) in the main producing areas, and it was likely to be the causal pathogen of hawthorn mosaic disease.
\end{abstract} 142 to 198 aa and at position 780 to 864 aa in the POL protein, respectively, between the hawthorn isolate and other isolates (apple, crabapple). A grafting test demonstrated that ApNMV was easily transmissible from
Keywords: RNA-seq, hawthorn tree, apple necrotic mosaic virus, new natural host, hawthorn mosaic disease
Ilarviruses, belonging to the Bromoviridae family, are worldwide viral pathogens that cause significant economic losses of many fruit trees such as apple, peach, plum, cherry, and apricot through direct or indirect reduction of yield and fruit quality (Pallás et al. 2012, 2013). Apple mosaic virus (ApMV), American plum line pattern virus, prune dwarf virus, and prunus necrotic ringspot virus (PNRSV) are the main pathogens infecting fruit trees (Pallás et al. 2012). These viruses may cause similar symptoms to some degree, which could be misleading in preliminary field diagnosis (Çağlayan et al. 2011; Desvignes 1999; Hammond 2011; Myrta et al. 2011; Pallás et al. 2012; Paunovic et al. 2011; Petrzik and Lenz 2011). Among these four ilarviruses, both ApMV and PNRSV can cause mosaic disease

${ }^{\dagger}$ Corresponding author: S. F. Li; sfli@ippcaas.cn

F. Xing and W. Y. Hou contributed equally to this work.

Funding: This work was supported by Key Inter-governmental Projects of the National Key Research and Development Program (2017YFE0110900), Fundamental Research Funds for Environment and Plant Protection Institute, CATAS (project number 1630042019017), Central Public-Interest Scientific Institution Basal Research Fund (no. Y2018PT67), and the program of China Scholarships Council (no. 201903250090).

*The $\boldsymbol{e}$-Xtra logo stands for "electronic extra" and indicates there are supplementary materials published online.

The author(s) declare no conflict of interest.

Accepted for publication 1 April 2020.

C 2020 The American Phytopathological Society in apple trees (Hu et al. 2016; Petrzik and Lenz 2011). In recent years, apple necrotic mosaic virus (ApNMV), a newly identified ilarvirus, also shows a high relationship with apple mosaic disease in apple trees in China (Xing et al. 2018).

ApNMV was first isolated from apple leaves infected with mosaic disease in Japan but occurs much less frequently in Japan (Noda et al. 2017). In contrast, ApNMV is widespread in apple-producing areas in China (Xing et al. 2018). Usually, apple leaves infected by ApNMV are found to exhibit obvious symptoms including chlorosis, pale yellow mosaic to bright cream-colored irregular spots, rings, or brownish necrotic spots. Bands and/or line patterns along the main veins were observed with uneven distribution on apple leaves (Noda et al. 2017; Xing et al. 2018).

The ApNMV genome consists of three single-stranded positive-sense RNA segments, namely, RNA1, RNA2, and RNA3 (Noda et al. 2017). RNA1 encodes a replicase protein containing a conserved methyltransferase/helicase (MET/HEL) domain (3,171 nucleotides [nt]; 1,056 amino acids [aa]). RNA2 is monocistronic and encodes a nonstructural protein, RNA polymerase (POL), of 855 to 867 aa, which contains the highly conserved motif sequence of glycine-aspartic acid-aspartic acid and plays an essential role in virus replication, and RNA3 contains two open reading frames (ORFs) encoding a movement protein (MP, 280 to $281 \mathrm{aa})$ at the $5^{\prime}$ terminal and a coat protein (CP, 219 aa) at the $3^{\prime}$ terminal, respectively (Noda et al. 2017; Xing et al. 2018). The dendrogram analysis indicated a close genetic relationship of ApNMV with ApMV and PNRSV. To date, ApNMV was also reported in apple trees in South Korea (Cho et al. 2017) and India (Nabi et al. 2020) and was detected in crabapple trees (Malus spp., family Rosaceae), an ornamental apple germplasm resource (Hu et al. 2019). 
Hawthorn (Crataegus spp., family Rosaceae) has an economic value for nutrition and medicine and is widely planted in China. However, until now, only apple chlorotic leaf spot virus, a latent virus in most commercial cultivars, was reported to be the main virus in hawthorn trees (Guo et al. 2016; Sweet 1980; Wang and Dai 2015). In this study, the high-throughput sequencing (HTS) of RNA, an unbiased technique allowing us to detect a broad range of viruses in plants (Nagano et al. 2015), was used to identify the viruses in hawthorn leaves showing mosaic symptoms. ApNMV tested positive in hawthorn trees, and its genome was sequenced and characterized. To the best of our knowledge, this is the first report of ApNMV in hawthorn trees, revealing hawthorn trees as a new natural host for ApNMV.

\section{Materials and Methods}

Plant materials and virus isolates. The hawthorn leaves were collected from the main hawthorn-producing provinces and regions in China, including Hebei, Jilin, Liaoning, Shandong, and Xinjiang. The collected samples were stored at low temperature using frozen blue ice and immediately frozen using liquid nitrogen at $-196^{\circ} \mathrm{C}$ upon arrival in the laboratory. They were further stored at $-80^{\circ} \mathrm{C}$ until total RNA was extracted.

Library preparation and RNA sequencing. A mixed library that contained two leaf samples from Xinjiang Province showing mosaic symptoms was constructed. Total RNA was extracted from the symptomatic leaves using TRIzol reagent (Tiangen, China). RNA degradation was monitored on $1 \%$ agarose gels. RNA purity was checked using the NanoPhotometer spectrophotometer (Implen, U.S.A.). RNA concentration was measured using a Qubit RNA Assay Kit in a Qubit 2.0 Fluorometer (Life Technologies, U.S.A.). RNA integrity was assessed using the RNA Nano 6000 Assay Kit of the Agilent Bioanalyzer 2100 system (Agilent Technologies, U.S.A.). Ribosomal RNA was removed by an Epicentre Ribo-Zero rRNA Removal Kit (Epicentre, U.S.A.), and mRNA from the tested plants was enriched using the poly-T attached to magnetic beads. The sequencing library was generated using the rRNA-depleted mRNA of total RNA with a NEBNext Ultra Directional RNA Library Prep Kit for Illumina (NEB, U.S.A.) following the manufacturer's recommendations. The library was sequenced using an Illumina HiSeq 4000 platform and generated 150 -bp paired-end reads.

Analysis and assembly of RNA-seq data. Raw reads from the Illumina platform were trimmed with the minimum quality of 25 , and then paired and duplicate reads were removed and the processed reads assembled de novo into larger contigs by the SPAdes assembler method with a k-mer of 32 using commercial software Geneious (https://www.geneious.com/). The final contigs were screened against the RefSeq genomes of viruses and viroids database from NCBI (https://www.ncbi.nlm.nih.gov/genome/viruses/) using the tblastx program in Geneious.

Total RNA extraction and reverse transcription (RT) of hawthorn leaf samples. Total RNA was extracted using an RNAprep Pure Plant Kit (TianGen, China) following the manufacturer's instructions and then treated with DNase I for $15 \mathrm{~min}$ at room temperature to remove DNA. The first-strand cDNAs were synthesized by RT reactions using M-MLV RT (Promega, China) with random hexamer primers according to the manufacturer's protocol.

Detection of ApNMV and apple stem pitting virus (ASPV) in hawthorn trees by RT-PCR. PCR was performed using the specific primer sets ApNMV-F/-R and ApN-F2/-R2 for the detection of ApNMV, as described before (Xing et al. 2018), and specific primer sets szASPV-F/R and szASPV-F2/R2 for ASPV. For the detection of ASPV, the master mix of samples was denatured at $94^{\circ} \mathrm{C}$ for 4 min and followed by 35 cycles of PCR ( 15 cycles of nested PCR) amplification with Taq polymerase (Bingda, China) at $94^{\circ} \mathrm{C}$ for $30 \mathrm{~s}, 55^{\circ} \mathrm{C}$ $\left(54^{\circ} \mathrm{C}\right.$ for the nested PCR) for $30 \mathrm{~s}$, and $72^{\circ} \mathrm{C}$ for $40 \mathrm{~s}$ ( $30 \mathrm{~s}$ for the nested PCR), with a final step of $10 \mathrm{~min}$ at $72^{\circ} \mathrm{C}$. PCR products were observed by $1 \%$ agarose gel electrophoresis using $1 \times$ TAE buffer (0.04 M Tris-acetate, $1 \mathrm{mM}$ EDTA), purified with a gel DNA purification kit (Axygen, China) and sequenced by Sangon Biotech (Shanghai) Co.
Cloning of the complete nucleotide sequences of ApNMV isolates from hawthorn trees. To obtain the complete nucleotide sequence of an ApNMV isolate from a hawthorn tree, Escherichia coli polymerase (NEB, China) was used to add poly A tails to $3^{\prime}$ ends of viral RNA1 to RNA3 molecules. M-MLV RT was used for the synthesis of first-strand cDNAs with a random hexamer primer. First, three primer sets (ApnS1/fullRNA1-R1, ApnS1/fullRNA1-R1, and RNA3mcF/fullRNA1-R1) were designed to amplify the partial genomes of ApNMV RNA1 to RNA3, respectively. The amplification of cDNA ends of ApNMV isolates were carried out using the SMARTer RACE $5^{\prime} / 3^{\prime}$ Kit (Clontech, U.S.A.) following the manufacturer's instructions using the primers 5R1GSP1/5R1GSP2, 5R2GSP1/5R2GSP2, and 5R3GSP1/5R3GSP2 for the $5^{\prime}$-end sequences and 3R1GSP1, 3RACE$\mathrm{R} 2-\mathrm{Ft}$, and $3 \mathrm{ApN}-\mathrm{RACE} 2$ for the 3 '-end sequences, respectively. Finally, the sequenced cDNA fragments were assembled, and complete nucleotide sequences of the three RNA segments of ApNMV from a hawthorn tree were obtained. All amplification reactions were performed using Q5 high-fidelity DNA polymerase (NEB, China). All primers used in this study are listed in Supplementary Table 1.

Phylogenetic analysis. Nucleotide sequences of ApNMV isolated from hawthorn trees based on the MET/HEL, POL, MP, and CP were aligned with the homologous sequences of other ApNMV isolates from GenBank using ClustalW, and the phylogenetic relationships of the four aligned ORF sequences were assessed by MEGA version 6 (Tamura et al. 2013) using the neighbor-joining method (Saitou and Nei 1987) with 1,000 bootstrap replications.

Transmission test of ApNMV by grafting. The hawthorn shoots that showed mosaic symptoms from Xinjiang Province were collected as virus sources. Transmission experiments of ApNMV were conducted by bud grafting from hawthorn trees to four 1-year-old and one 3-year-old apple plantlets, which were free of ApNMV. The plants were maintained at about $25^{\circ} \mathrm{C}$ in the greenhouse with $16-\mathrm{h} /$ 8-h (day/night) photoperiods. The symptoms on the tested plants were under continuous observation, and RT-PCR together with Sanger dideoxy sequencing was used to confirm the infection of ApNMV on these grafting-transmitted plants.

\section{Results}

Symptoms and occurrence of mosaic disease on hawthorn trees in China. A survey on symptoms and occurrence of mosaic disease on hawthorn trees was conducted in the main hawthornproducing provinces and regions (such as Hebei, Jilin, Liaoning, Shandong, and Xinjiang) of China. A total of 11,685 hawthorn trees were surveyed, and only six trees (2/10,000 in Chengde, Hebei; $1 /$ 135 in Gongzhuling, Jilin; 1/1,500 in Shenyang, Liaoning; 0/20 in Linyi, Shandong; and 2/30 in Luntai, Xinjiang) showed typical mosaic symptoms. The mosaic symptoms in hawthorn trees varied from mild, moderate, to severe chlorosis and could include yellowing, pale yellow mosaic to bright cream-colored irregular spots, and rings. Bands and/or line patterns were observed with uneven distribution on hawthorn leaves (Fig. 1A to D). The symptoms are often restricted to parts of the whole hawthorn trees. The symptoms of mosaic disease on hawthorn trees were similar to those on apple leaves caused by ApNMV, so we speculated a crucial role of ApNMV in causing mosaic disease on hawthorn trees. In addition, other symptoms were also found, including chlorosis, yellowing, or necrosis on the leaf margins, but they were obviously different from the typical symptoms of mosaic disease (Fig. 1E to G).

RNA-seq analysis of hawthorn leaves with mosaic symptoms. In total, 43,252,990 clean reads that had passed the quality control were produced after having been trimmed, paired, and merged. The assembly generated 171,338 contigs ranging from 55 to 45,814 nt. Three contigs of 3,357, 2,748, and 1,934 nt, respectively, were found to share high nucleotide and amino acid similarity with ApNMV, and an additional two contigs of 9,310 and 1,474 nt had high identities with ASPV. Using BLAST against the viral sequences in the GenBank Virus Reference Database (http://www.ncbi.nlm. nih.gov), the identities of the three contigs with ApNMV were observed (96.9\% [RNA1], 95.6\% [RNA2], and 94.5\% [RNA3]), and they covered the genome sequence of ApNMV available from NCBI 
on $100 \%$ (RNA1), 100\% (RNA2), and 99\% (RNA3), respectively. Meanwhile, one contig of 9,310 nt shared $81.6 \%$ identity with ASPV on $99 \%$ coverage, and the other of 1,934 nt showed $90.8 \%$ nucleotide identity with ASPV on 59\% coverage.

Complete nucleotide sequence and genome characteristics of ApNMV isolated from detected hawthorn tree. To confirm the HTS result and to understand the differences of ApNMV isolates from hawthorn and apple trees, we cloned and sequenced the complete nucleotide sequences of ApNMV isolated from the same hawthorn tree with mosaic symptoms from Xinjiang Province. Its genome consisted of three RNA molecules with sizes of 3,378 nt (RNA1, accession no. MN656198), 2,778 nt (RNA2, accession no. MN656199), and 1,917 nt (RNA3, accession no. MN656200). The nucleotide sequence identities of RNA1 to RNA3 obtained from hawthorn trees, apple trees, and crabapple trees were 93.8 to $96.8 \%, 89.7$ to $96.1 \%$, and 89.8 to $94.6 \%$, respectively, as shown in Table 1. The identities of MET/HEL, POL, MP, and CP of ApNMV isolated from the hawthorn tree with other isolates were 93.7 to $96.9 \%, 89.5$ to $96.1 \%, 88.4$ to $94.5 \%$, and 91.8 to $96.8 \%$, respectively, based on the nucleotide sequences, and were 97.8 to $98.7 \%, 92.1$ to $96.8 \%, 90.4$ to $94.6 \%$, and 94.5 to $97.3 \%$ based on the amino acid sequences, respectively. The MET/HEL was highly conserved, whereas the POL and MP showed great variation among the four proteins. Two hypervariable regions were found at position 142 to 198 aa and at position 780 to 864 aa in the POL encoded by RNA2, which shared 59.2 to $85.7 \%$ and 64.0 to $89.3 \%$ sequence identities between the hawthorn isolate and other isolates (apple and crabapple), respectively (Fig. 2).
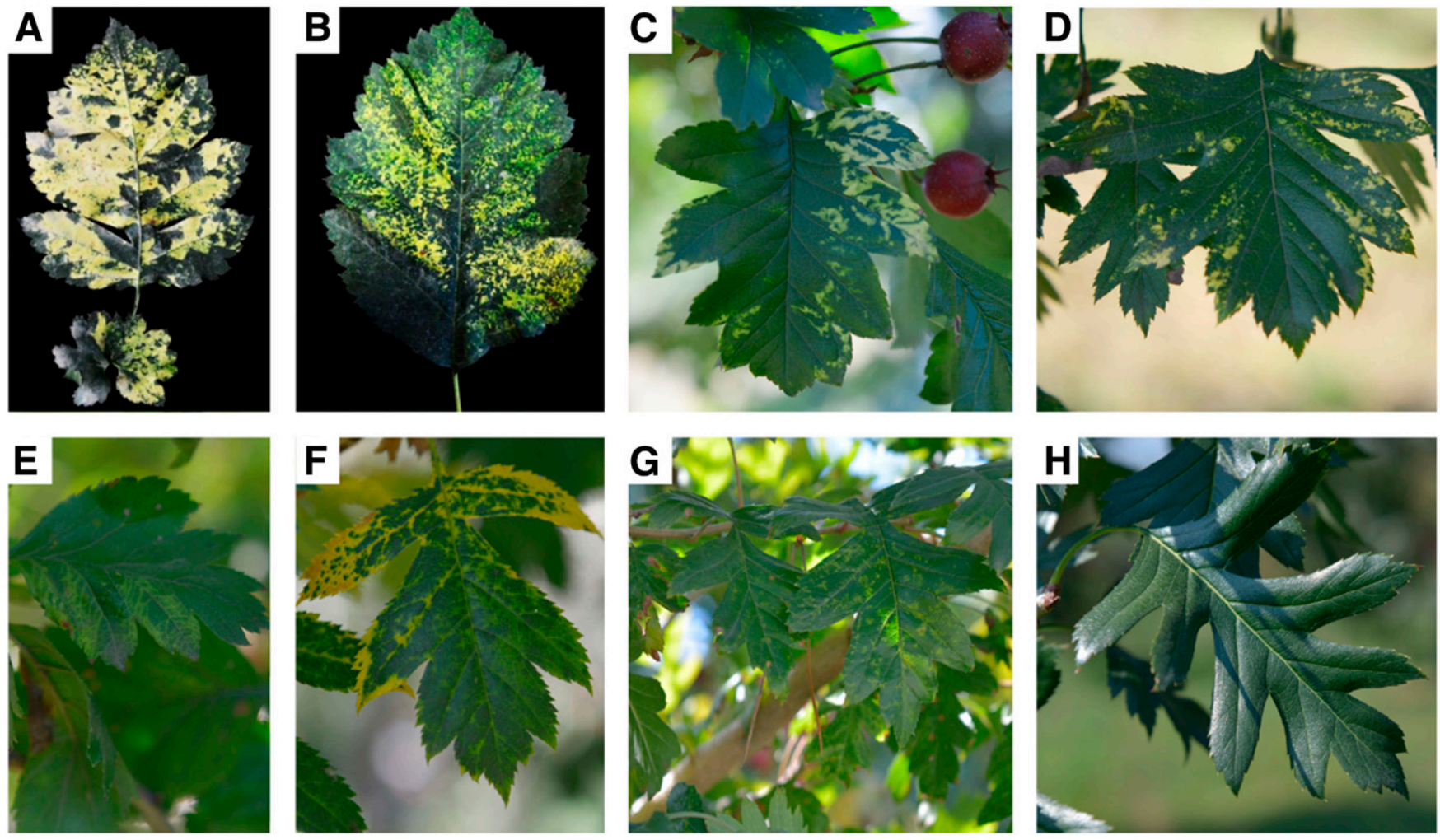

Fig. 1. The investigated symptoms on hawthorn trees in the fields: $\mathbf{A}$ to $\mathbf{D}$, typical symptoms caused by mosaic diseases with yellowing, mosaic, line pattern, pale yellow mosaic to bright cream-colored irregular spots, and rings; $\mathbf{E}$ to $\mathbf{G}$, other symptoms, including chlorosis, yellowing, or spots on the leaves but obviously different from the typical symptoms of mosaic disease; and $\mathbf{H}$, asymptomatic hawthorn leaf.

Table 1. The sequence identities of apple necrotic mosaic virus (ApNMV) isolated from hawthorn tree with others at nucleotide and amino acid level ${ }^{\mathrm{a}}$

\begin{tabular}{|c|c|c|c|c|c|c|c|c|c|c|c|c|}
\hline \multirow[b]{3}{*}{ Isolate $^{b}$} & \multirow[b]{3}{*}{ Host plant } & \multicolumn{3}{|c|}{ RNA1 (\%) } & \multicolumn{3}{|c|}{ RNA2 (\%) } & \multicolumn{5}{|c|}{ RNA3 (\%) } \\
\hline & & \multirow[b]{2}{*}{ Full length } & \multicolumn{2}{|c|}{ MET/HEL } & \multirow[b]{2}{*}{ Full length } & \multicolumn{2}{|c|}{ POL } & \multirow[b]{2}{*}{ Full length } & \multicolumn{2}{|c|}{ MP } & \multicolumn{2}{|c|}{ CP } \\
\hline & & & nt & $\overline{\mathbf{a a}}$ & & nt & aa & & nt & aa & nt & aa \\
\hline Hai & Crabapple & 96.3 & 96.4 & 98.3 & 93.6 & 93.4 & 96.5 & 91.5 & 89.0 & 90.4 & 95.9 & 95.0 \\
\hline Hua & Apple & 93.8 & 93.7 & 98.3 & 95.0 & 94.9 & 95.9 & 90.4 & 89.2 & 91.8 & 94.2 & 95.0 \\
\hline $\mathrm{Qu}$ & Apple & 96.7 & 96.7 & 98.6 & 96.1 & 96.1 & 96.2 & 94.6 & 94.1 & 93.9 & 96.8 & 95.4 \\
\hline P129 & Apple & 96.0 & 96.1 & 97.9 & 95.1 & 95.0 & 95.7 & 93.5 & 92.6 & 92.9 & 95.5 & 97.3 \\
\hline AM27 & Apple & 95.2 & 95.2 & 97.9 & 90.8 & 90.8 & 93.0 & 89.8 & 88.4 & 91.1 & 94.4 & 95.4 \\
\hline AM36 & Apple & 95.1 & 95.2 & 98.0 & 90.7 & 90.7 & 93.0 & 90.2 & 88.5 & 91.4 & 94.5 & 95.4 \\
\hline AM72 & Apple & 96.6 & 96.6 & 98.2 & 89.7 & 89.5 & 92.1 & 89.8 & 89.7 & 91.8 & 91.8 & 95.4 \\
\hline AM75 & Apple & 96.8 & 96.9 & 98.7 & 96.0 & 95.9 & 96.8 & 94.5 & 94.2 & 94.6 & 96.5 & 96.3 \\
\hline AM95 & Apple & 95.5 & 95.5 & 97.9 & 90.6 & 90.4 & 93.1 & 90.0 & 88.6 & 91.8 & 93.8 & 94.5 \\
\hline AM125 & Apple & 96.8 & 96.8 & 97.8 & 96.1 & 96.0 & 96.6 & 94.2 & 94.5 & 93.6 & 95.8 & 95.9 \\
\hline
\end{tabular}

a MET/HEL = methyltransferase/NTP-binding helicase; $\mathrm{POL}=$ RNA polymerase; $\mathrm{MP}=$ movement protein $; \mathrm{CP}=$ coat protein; $\mathrm{nt}=$ the sequence alignment at nucleotide level; and aa = the sequence alignment at amino acid level. The highest and the lowest are indicated in bold type.

b The accession numbers of RNA1, RNA2, and RNA3 of ApNMV isolates used in this study are Hai (MG924894, MG924897, and MG924900), Hua (MG924895, MG924898, and MG924901), Qu (MG924896, MG924899, and MG924902), P129 (LC108993 to LC108995), AM27 (KY808376 to KY808378), AM36 (KY808379 to KY808381), AM72 (KY808382 to KY808384), AM75 (KY808385 to KY808387), AM95 (KY808388 to KY808390), and AM125 (KY808391 to KY808393), respectively. 


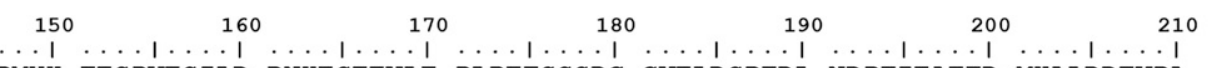

SZ POL 141 EHN-DVPMYH TESPVESILD RNHESEEVLT PLPTTSSSDC GVELDSPTDL NDPTIEAETD MHALDDEVPA 209

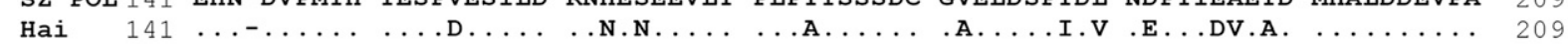

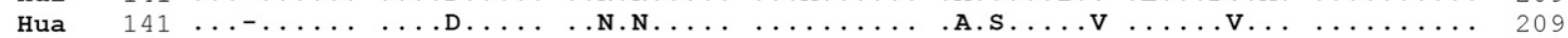

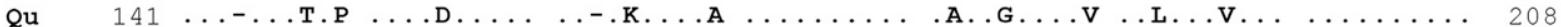

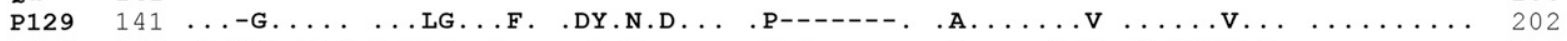

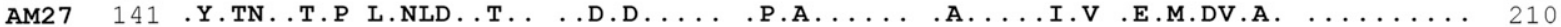

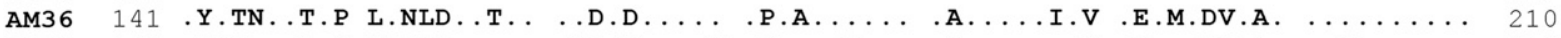

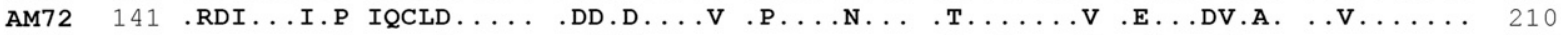

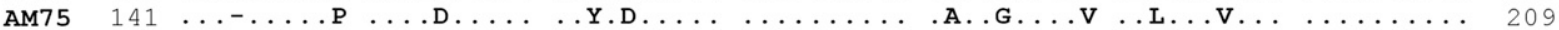

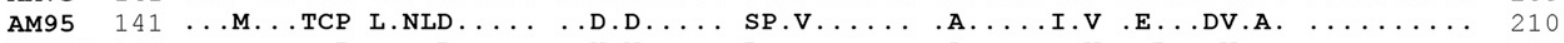

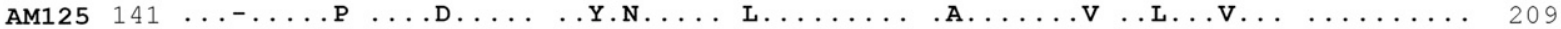

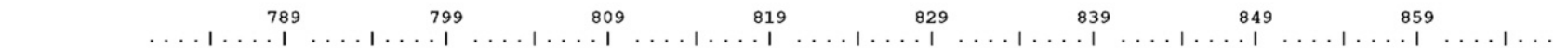

SZ POL 780 GDEVRAQRGK TDMSKTPRRW ADVVEHTESP NRDYSDERIT RSDLIPIYGE GK-FLSPVNR SRRPSKKNRS IAKKTGKVEL NTTEPR-- 864

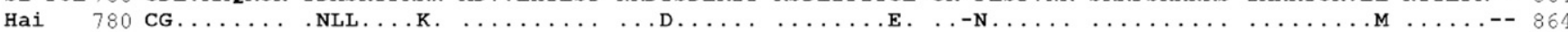

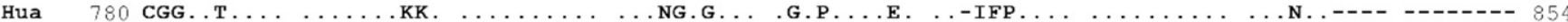

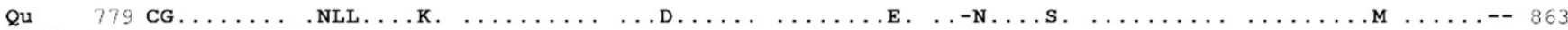

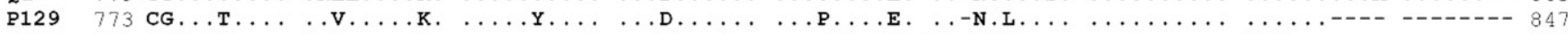

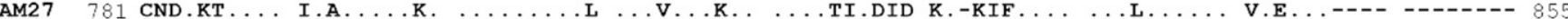

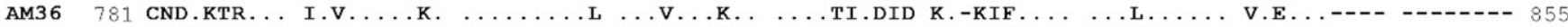

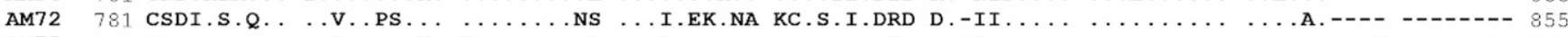

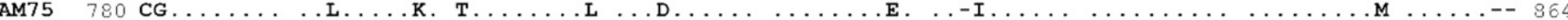

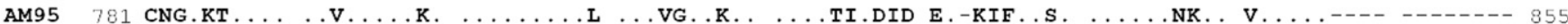

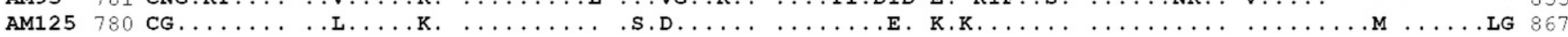

Fig. 2. Two hypervariable regions in the RNA polymerase (POL) protein among apple necrotic mosaic virus isolates. SZ POL was obtained in this study. It showed 59.2 to $85.7 \%$ and 64.0 to $89.3 \%$ sequence identities at position 142 to 198 aa and at position 780 to 864 aa, respectively, between the hawthorn isolate and other isolates (apple, crabapple).
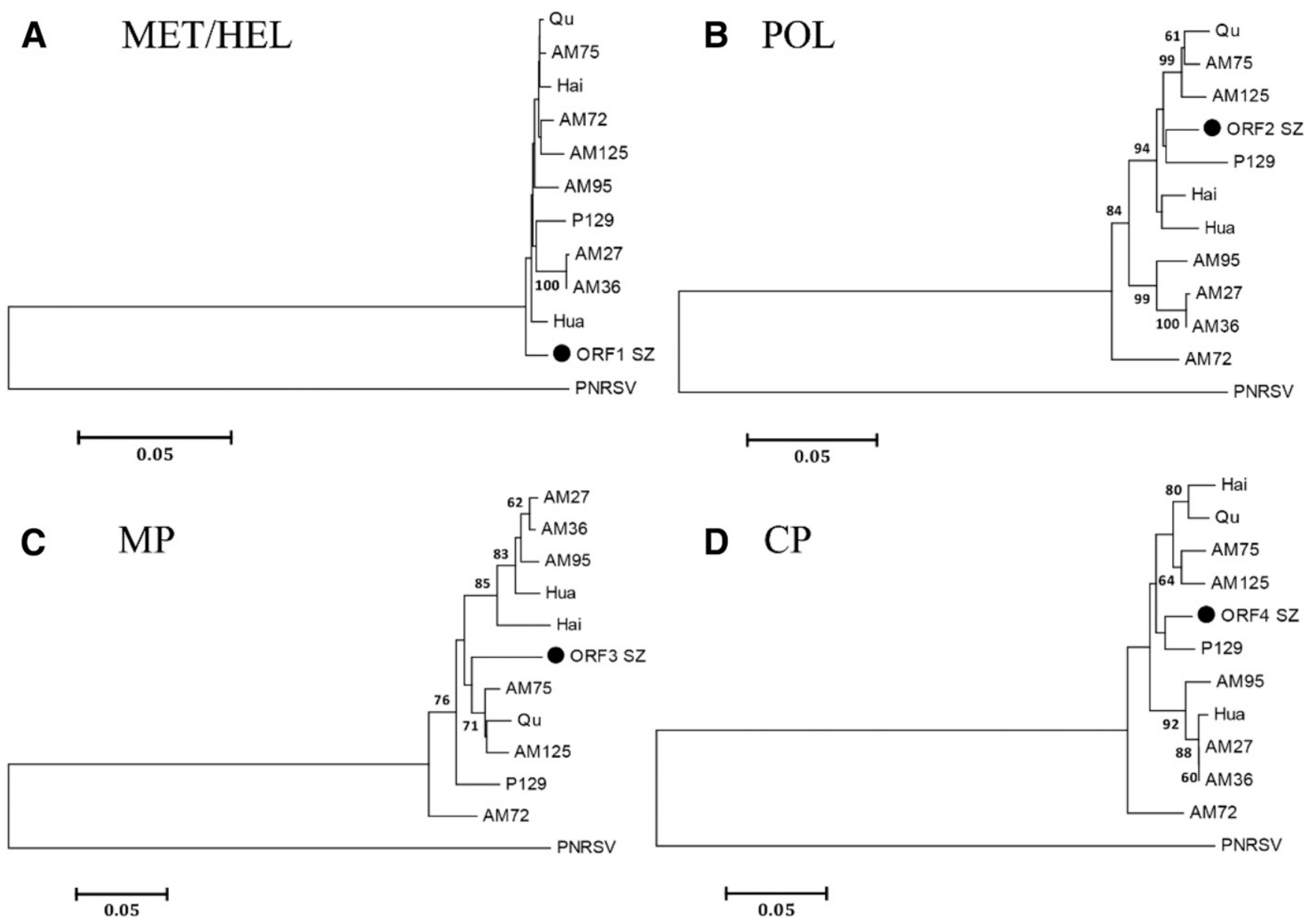

Fig. 3. Phylogenetic relationship of the apple necrotic mosaic virus (ApNMV) hawthorn isolate with other isolates from apples and crabapples. The tree was constructed using the neighbor-joining method with 1,000 bootstrap replications based on the amino acids of $\mathbf{A}$, methyltransferase/NTP-binding helicase (MET/HEL); $\mathbf{B}, \mathrm{RNA}$ polymerase (POL); $\mathbf{C}$, movement protein (MP); and D, coat protein (CP). Prunus necrotic ringspot virus (PNRSV) (accession nos. NC004362, NC004363, and NC004364) was chosen as an outgroup. Only bootstrap values $\geq 60 \%$ are shown. 
After the complete viral genome was obtained, clean reads were mapped to the ApNMV genome, indicating a nearly whole coverage (Supplementary Fig. 1). The nucleotide identity percentage values were $99.7 \%$ (RNA1), 99.0\% (RNA2), and 97.9\% (RNA3) between the sequence obtained with HTS and the individual clones obtained by $5^{\prime}-3^{\prime}$ RACE and RT-PCR, respectively, which showed the ApNMV variability in the infected tissue.

Phylogenetic analysis of ApNMV from hawthorn trees with other isolates. Phylogenetic analysis of the ApNMV hawthorn isolate with others isolated from crabapple and apple trees was performed using the neighbor-joining method on the selected amino acid sequences of the MET/HEL, POL, MP, and CP genes. Results showed the ApNMV hawthorn isolate had a closer genetic relationship with P129 isolate based on the POL and CP, whereas it seemed to be more closely related to AM75 and Qu isolates based on MET/ HEL and MP (Fig. 3), consistent with phylogenetic relationship constructed based on the nucleotide sequences or using the maximum likelihood method (data not shown). But no host- or geographyrelated conclusion could be drawn from the phylogenetic analysis.

Transmission test of ApNMV by grafting. Four 1-year-old and one 3-year-old apple plantlets, which had been confirmed as virusfree plantlets via tissue culture with the meristem, were grafted using hawthorn buds infected by ApNMV. At 24 days postgrafting, new leaves on the top of the five tested apple plantlets were found to present symptoms of chlorosis, yellowing, mosaic, curling, and necrosis (Fig. 4A). All of the five grafted plantlets were positive for ApNMV, as confirmed by RT-PCR (Fig. 4B). It suggested that ApNMV could be easily transmitted from hawthorn to apple by grafting.
Occurrence and distribution of ApNMV and ASPV in hawthorn trees in China. In the present study, we confirmed hawthorn trees as a new natural host for ApNMV. To understand the occurrence and distribution of ApNMV in the main hawthorn-producing provinces and regions in China, a total of 145 leaf samples were collected from provinces or regions of Hebei, Jilin, Liaoning, Shandong, and Xinjiang. ApNMV was detected by RT-PCR and nested PCR, as described previously (Xing et al. 2018). A total of 20 samples (13.8\%) tested positive for ApNMV (Table 2). Six samples showing typically mosaic symptoms were found infected by ApNMV, indicating a correlation with the presence of ApNMV and the mosaic symptoms on hawthorn trees. ApNMV also tested positive with a low percentage in asymptomatic (i.e., 5.9\%, four out of 68, Fig. $1 \mathrm{H}$ ) and other-symptomatic (i.e., $14.1 \%, 10$ out of 71, Fig. 1E to G) hawthorn samples. In addition, ASPV was found positive with a low frequency (i.e., $2.1 \%$, three [two mosaic leaf samples from Xinjiang and one asymptomatic leaf sample from Shenyang] out of 145), suggesting a lower relationship with mosaic disease. Taken together, the data suggested that ApNMV might be the causative agent of the hawthorn mosaic disease other than ASPV.

\section{Discussion}

The viral diseases of hawthorns are not well characterized (Guo et al. 2016; Wang and Dai 2015), most probably due to its lower economic importance compared with apple or pear. In a previous study, we identified the association between ApNMV presence and apple mosaic disease (Xing et al. 2018). In the present study, we collected hawthorn leaves with mosaic symptoms similar to those observed on

A
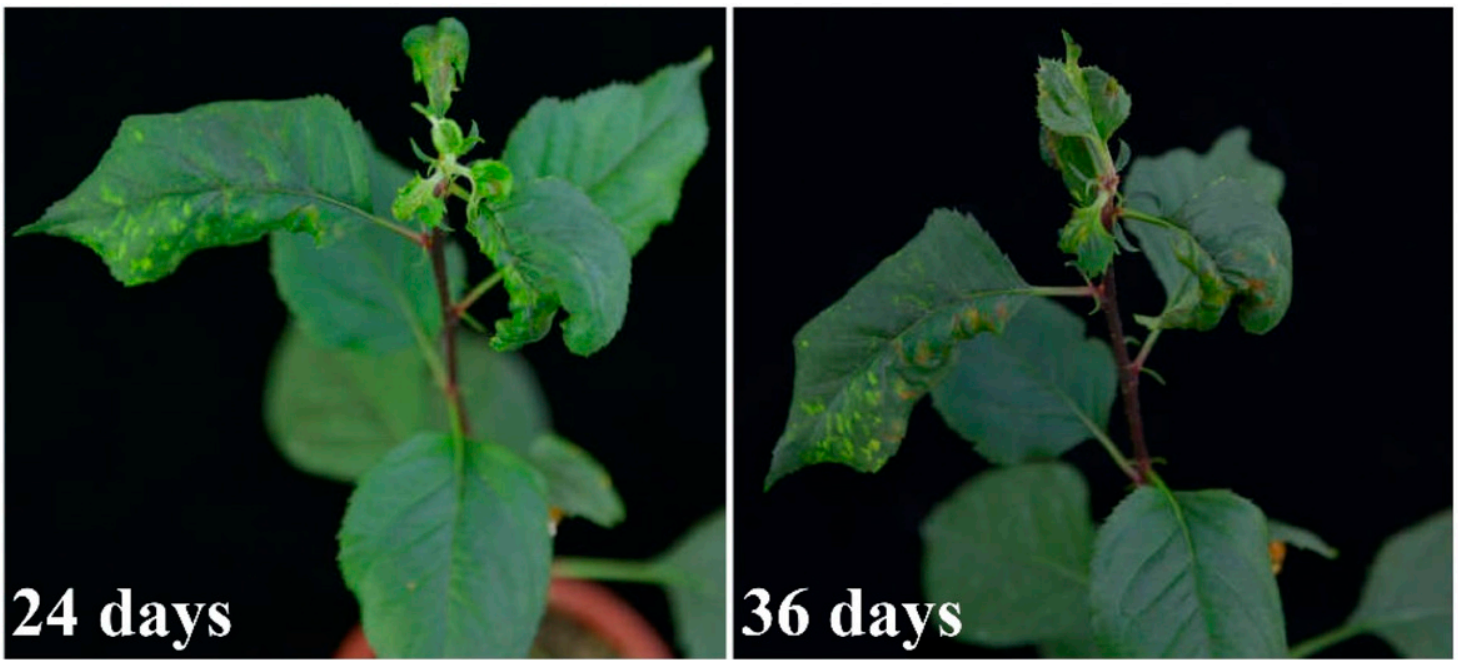

B grafted apple plantlets

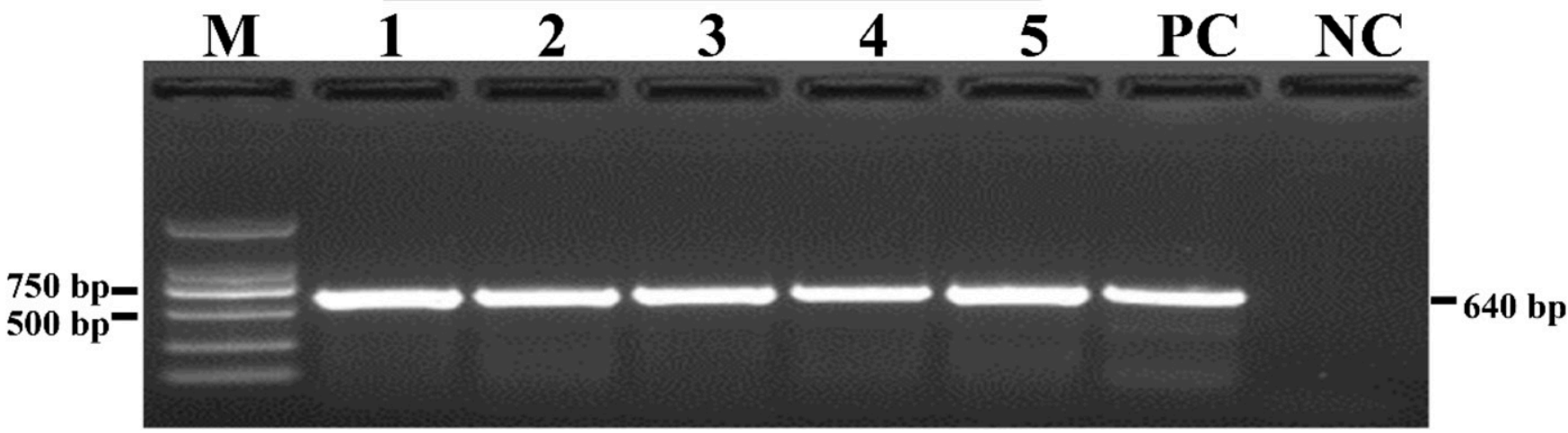

Fig. 4. Transmission test of apple necrotic mosaic virus (ApNMV) by grafting. A, The new leaves on the top of apple plantlets presented obvious symptoms with chlorosis, yellowing, mosaic, curling, and necrosis at 24 and 36 days postgrafting. B, ApNMV infection was confirmed at 24 days postgrafting by RT-PCR. M = DNA marker 2000; PC = an apple tree infected by ApNMV as the positive control; and NC = double-distilled water as the negative control. 
Table 2. Geographical distribution and incidence of apple necrotic mosaic virus from hawthorn trees in the major hawthorn-producing areas in China ${ }^{\mathrm{a}}$

\begin{tabular}{|c|c|c|c|c|c|c|}
\hline \multirow[b]{2}{*}{ Location } & \multicolumn{2}{|c|}{ Asymptomatic } & \multicolumn{2}{|c|}{ Typically mosaic } & \multicolumn{2}{|c|}{ Other symptoms } \\
\hline & No. of samples & Positive (rate \%) & No. of samples & Positive (rate \%) & No. of samples & Positive (rate \%) \\
\hline Hebei & 6 & $1(16.7)$ & 2 & $2(100)$ & 28 & $7(25)$ \\
\hline Jilin & 16 & $1(6.3)$ & 1 & $1(100)$ & 19 & $1(5.3)$ \\
\hline Liaoning & 11 & $0(0)$ & 1 & $1(100)$ & 24 & $2(8.3)$ \\
\hline Shandong & 20 & $0(0)$ & $\ldots$ & $\ldots$ & $\ldots$ & $\ldots$ \\
\hline Xinjiang & 15 & $2(13.3)$ & 2 & $2(100)$ & $\ldots$ & $\ldots$ \\
\hline Total & 68 & $4(5.9)$ & 6 & $6(100)$ & 71 & $10(14.1)$ \\
\hline
\end{tabular}

a Typically mosaic symptoms refers to mosaic symptoms in hawthorn trees that varied from mild, moderate, to severe chlorosis and could include yellowing, pale yellow mosaic to bright cream-colored irregular spots, rings, or irregular bands and/or line patterns on hawthorn leaves; other symptoms included chlorosis, yellowing, or necrosis on the leaf margins, which might be caused by low temperature, bacterial diseases, or fungal diseases and were significantly different from the typically mosaic symptoms.

apple trees. By HTS, ApNMV was detected for the first time on hawthorns and might be the causing pathogen of hawthorn mosaic disease. The results, confirmed by RT-PCR, demonstrate that hawthorn is a new natural host for ApNMV. Nevertheless, to further confirm ApNMV as the causal agent of hawthorn mosaic disease, inoculation of virus-free hawthorn plantlets with an infectious clone of ApNMV hawthorn isolate to display the resulting mosaic symptom is necessary in future.

The complete genome of ApNMV isolate from a hawthorn tree was cloned and sequenced. Multiple sequence alignment and phylogenetic analysis demonstrated the genome of ApNMV isolated from the hawthorn had a relatively high sequence identity to those from apple and crabapple trees at both nucleotide and amino acid levels. However, two hypervariable regions were found at $\mathrm{N}$ - and $\mathrm{C}$ terminals in the POL protein encoded by RNA2, respectively. It is possible the hypervariable regions are associated with adaptability to the different hosts, but this hypothesis still remains to be further studied. Simultaneously, the high sequence similarity among the isolates might support transmission of ApNMV between hawthorn and apple trees. Our grafting test proved that ApNMV could be easily transmitted from hawthorn trees to apple trees and then caused severe mosaic, distortions, and necrosis on apple trees.

In addition, geographical distribution and incidence of ApNMV were also determined from hawthorn trees in some major hawthorn-producing areas in China. The presence of ApNMV in all six samples with mosaic symptoms indicated that ApNMV was associated with hawthorn mosaic disease. ApNMV was also detected in a low incidence rate of the hawthorn trees with other symptoms $(14.1 \%)$ or without symptoms $(5.9 \%)$. As for other fruit tree viruses (Pallás et al. 2012), it might suggest that symptom development is a complex phenomenon influenced by the cultivar, growth vigor, season (temperature, light intensity), virus isolate, virus titer, and so on. This is consistent with our survey that only six out of 11,685 hawthorn trees with mosaic symptoms were observed. These results indicated ApNMV might not be widespread on hawthorn trees in China. It might be related to low growing areas and low frequency of resource exchange in the hawthorn industry. Indeed, ApNMV infection does not seem to spread so much in the hawthorn population. However, the hawthorn planting areas are usually close to the appleplanting areas in northern China according to our surveys, so there is a nonnegligible risk to transmit ApNMV from hawthorn to apple trees when using the same pruning tools. In this situation, it will be a potential threat affecting the economic cultivation of apples.

In our study, ASPV, a latent virus in most commercial cultivars (Jelkmann and Paunovic 2011), was also identified and confirmed using HTS together with RT-PCR. However, only three (two mosaic and one asymptomatic) out of 145 leaf samples tested positive for ASPV, indicating a lower incidence and lower association with mosaic disease. Although apple stem pitting disease (ASPD) was reported in Crataegus monogyna about 40 years ago when the causal agent of ASPD was not yet identified as ASPV (Sutic et al. 1999; Sweet and Campbell 1976), our results provide, for the first time, definite evidence that hawthorn trees could be infected with ASPV in natural conditions.
In summary, a new natural host, hawthorn, has been discovered for ApNMV, highly associated with hawthorn mosaic disease. High genomic similarity of the identified genomes with ApNMV genomes from apple and crabapple, suggesting potential transmission of ApNMV among hawthorns and apples, although this hypothesis warrants further investigation. However, ApNMV was detected with a low incidence from hawthorn trees in the major hawthornproducing areas in China. This was the first report of ApNMV from hawthorn trees. It will be helpful to develop the diagnostic protocol and disease-control measures of ApNMV in hawthorn trees and lay a foundation for the in-depth unraveling of the molecular mechanism of mosaic disease caused by ApNMV.

\section{Acknowledgments}

We thank Prof. Hailou Zhang (Liaoning Academy of Agricultural Sciences, Liaoning, China), Prof. Wenxuan Dong (Shenyang Agricultural University, Liaoning, China), Dr. Xiao Zhang (Shenyang Agricultural University, Liaoning, China), and Prof. Hongwei Song (Jilin Academy of Agricultural Sciences, Jilin, China) for their help with sampling of hawthorn leaves.

\section{Literature Cited}

Çağlayan, K., Ulubas Serce, C., Gazel, M., and Varveri, C. 2011. Prune dwarf virus. Pages 199-205 in: Virus and Virus-Like Diseases of Pome and Stone Fruits. A. Hadidi, M. Barba, T. Candresse, and W. Jelkmann, eds. American Phytopathological Society, St. Paul, MN.

Cho, I. S., Kwon, S. J., Yoon, J. Y., Chung, B. N., Hammond, J., and Lim, H. S. 2017. First report of apple necrotic mosaic virus infecting apple trees in Korea. J. Plant Pathol. 99:815.

Desvignes, J. C. 1999. Virus Diseases of Fruit Trees. Centre Technique Intraprofessionnel des Fruits et Legumes, Paris, France.

Guo, W., Zheng, W., Wang, M., Li, X., Ma, Y., and Dai, H. Y. 2016. Genome sequences of three apple chlorotic leaf spot virus isolates from hawthorns in China. PLoS One 11:e0161099.

Hammond, R. W. 2011. Prunus necrotic ringspot virus. Pages 207-213 in: Virus and Virus-Like Diseases of Pome and Stone Fruits. A. Hadidi, M. Barba, T Candresse, and W. Jelkmann, eds. American Phytopathological Society, St. Paul, MN.

Hu, G. J., Dong, Y. F., Zhang, Z. P., Fan, X. D., and Ren, F. 2019. Molecular characterization of apple necrotic mosaic virus identified in crabapple (Malus spp.) tree of China. J. Integr. Agric. 18:698-701.

Hu, G. J., Dong, Y. F., Zhang, Z. P., Fan, X. D., Ren, F., Li, Z. N., and Zhou, J. 2016. First report of Prunus necrotic ringspot virus infection of apple in China. Plant Dis. 100:1955.

Jelkmann, W., and Paunovic, S. 2011. Apple stem pitting virus. Pages 35-40 in: Virus and Virus-Like Diseases of Pome and Stone Fruits. A. Hadidi, M Barba, T. Candresse, and W. Jelkmann, eds. American Phytopathological Society, St. Paul, MN.

Myrta, A., Herranz, M. C., Choueiri, E., and Pallas, V. 2011. American plum line pattern virus. Pages 181-183 in: Virus and Virus-Like Diseases of Pome and Stone Fruits. A. Hadidi, M. Barba, T. Candresse, and W. Jelkmann, eds. American Phytopathological Society, St. Paul, MN.

Nabi, S. U., Baranwal, V. K., Yadav, M. K., and Rao, G. P. 2020. Association of Apple necrotic mosaic virus (ApNMV) with mosaic disease in commercially grown cultivars of apple (Malus domestica Borkh) in India. 3 Biotech. 10: 122.

Nagano, A. J., Honjo, M. N., Mihara, M., Sato, M., and Kudoh, H. 2015 Detection of plant viruses in natural environments by using RNA-seq. Pages 89-98 in: Plant Virology Protocols. Methods in Molecular Biology (Methods and Protocols). I. Uyeda and C. Masuta, eds. Humana Press, New York, NY. 
Noda, H., Yamagishi, N., Yaegashi, H., Xing, F., Xie, J. P., Li, S. F., Zhou, T., Ito, T., and Yoshikawa, N. 2017. Apple necrotic mosaic virus, a novel ilarvirus from mosaic-diseased apple trees in Japan and China. J. Gen. Plant Pathol. 83:83-90.

Pallás, V., Aparicio, F., Herranz, M. C., Amari, K., Sanchez-Pina, M. A., Myrta, A., and Sanchez-Navarro, J. A. 2012. Ilarviruses of Prunus spp.: A continued concern for fruit trees. Phytopathology 102:1108-1120.

Pallás, V., Aparicio, F., Herranz, M. C., Sanchez-Navarro, J. A., and Scott, S. W. 2013. The molecular biology of ilarviruses. Adv. Virus Res. 87:139-181.

Paunovic, S., Pasquini, S., and Barba, M. 2011. Apple mosaic virus in stone fruits. Pages 91-95 in: Virus and Virus-Like Diseases of Pome and Stone Fruits. A. Hadidi, M. Barba, T. Candresse, and W. Jelkmann, eds. American Phytopathological Society, St. Paul, MN.

Petrzik, K., and Lenz, O. 2011. Apple mosaic virus in pome fruits. Pages 25-28 in: Virus and Virus-Like Diseases of Pome and Stone Fruits. A. Hadidi, M. Barba, T. Candresse, and W. Jelkmann, eds. American Phytopathological Society, St. Paul, MN

Saitou, N., and Nei, M. 1987. The neighbor-joining method: A new method for reconstructing phylogenetic trees. Mol. Biol. Evol. 4:406-425.
Sutic, D. D., Ford, R. E., and Tosic, M. T. 1999. Virus diseases of fruit trees. Pages 321-432 in: Handbook of Plant Virus Diseases. D. D. Sutic, R. E. Ford, and M. T. Tosic, eds. CRC Press, Boca Raton, FL.

Sweet, J. B. 1980. Hedgerow hawthorn (Crataegus spp.) and blackthorn (Prunus spinosa) as hosts of fruit tree viruses in Britain. Ann. Appl. Biol. 94:83-90.

Sweet, J. B., and Campbell, A. I. 1976. Pome fruit virus infections of some woody ornamental and indigenous species of Rosaceae. J. Hortic. Sci. 51:91-97.

Tamura, K., Stecher, G., Peterson, D., Filipski, A., and Kumar, S. 2013. MEGA6: Molecular Evolutionary Genetics Analysis version 6.0. Mol. Biol. Evol. 30: 2725-2729.

Wang, M., and Dai, H. 2015. First report of apple chlorotic leaf spot virus in hawthorn in China. Plant Dis. 99:164.

Xing, F., Robe, B. L., Zhang, Z. X., Wang, H. Q., and Li, S. F. 2018. Genomic analysis, sequence diversity, and occurrence of apple necrotic mosaic virus, a novel ilarvirus associated with mosaic disease of apple trees in China. Plant Dis. 102:1841-1847. 